\title{
ERRATUM
}

\section{Overview of guide RNA design tools for CRISPR-Cas9 genome editing technology}

\author{
Lihua Julie Zhu ( $ه)$
}

Department of Molecular, Cell and Cancer Biology, Program in Bioinformatics and Integrated Biology, Program in Molecular Medicine, University of Massachusetts Medical School, Worcester, MA 01605, USA

(C) Higher Education Press and Springer-Verlag Berlin Heidelberg 2015

Erratum to: Front. Biol. 2015, 10(4): 289, DOI: 10.1007/s11515-015-1366-y

The original version of this article unfortunately contained a mistake. The presentation of Table 1 was incorrect. The corrected table is given below.

Table 1 Overview of several representative gRNA design tools, that are available at: ${ }^{1} \mathrm{http}: / / \mathrm{www}$. bioconductor.org/ packages/release/bioc/html/CRISPRseek.html; ${ }^{2} \mathrm{http} / /$ crispr.mit.edu; ${ }^{3} \mathrm{http}: / /$ www.e-crisp.org/E-CRISP/; ${ }^{4} \mathrm{http}: / /$ cas9.cbi.pku. edu.cn, ${ }^{5} \mathrm{http}: / / \mathrm{www}$. multicrispr.net, and ${ }^{6} \mathrm{http}: / / \mathrm{www}$. broadinstitute.org/rnai/public/analysis-tools/sgrna-design.

\begin{tabular}{|c|c|c|c|c|c|c|}
\hline $\begin{array}{l}\text { Functions/ } \\
\text { Limits }\end{array}$ & CRISPRseek $^{1}$ & Zhang $\mathrm{Lab}^{2}$ & $\mathrm{eCRISP}^{3}$ & Kong $\mathrm{Lab}^{4}$ & $\begin{array}{l}\text { CRISPR } \\
\text { MultiTargeter }^{5}\end{array}$ & sgRNA Designer ${ }^{6}$ \\
\hline Off-target analysis & $\begin{array}{l}\text { Yes with } \\
\text { PDMM penalty scoring }\end{array}$ & $\begin{array}{l}\text { Yes with PDMM } \\
\text { penalty scoring }\end{array}$ & $\begin{array}{l}\text { Yes without } \\
\text { scoring }\end{array}$ & $\begin{array}{l}\text { Yes without } \\
\text { scoring }\end{array}$ & $\begin{array}{l}\text { Yes without } \\
\text { scoring }\end{array}$ & No \\
\hline gRNA efficacy prediction & Yes & No & No & No & No & Yes \\
\hline Batch search & Yes & Yes & Yes & No & No & No \\
\hline Paired configuration & $\begin{array}{l}\text { Nickase, } \\
\text { dCas9-Fok1 and } \\
\text { Cas9-DBD } \\
\text { fusion (dev) }\end{array}$ & Nickase & No & No & No & No \\
\hline Cas9 type & Flexible & SpCas9 Only & SpCas9 Only & SpCas9 Only & SpCas9 Only & SpCas9 Only \\
\hline $\begin{array}{l}\text { Alternative scoring matrix } \\
\text { for efficacy and off-targets }\end{array}$ & xYes & No & No & No & No & No \\
\hline Target species & Any & A specified set & A specified set & A specified set & & Human and mouse \\
\hline Restriction enzyme sites & Yes & No & No & No & No & No \\
\hline Compare set of sequences & Yes & No & No & No & Yes & No \\
\hline Predict secondary structure & Yes & No & No & Yes & No & No \\
\hline
\end{tabular}

Received June 21, 2015; accepted July 8, 2015

Correspondence: Lihua Julie Zhu

E-mail: Julie.zhu@umassmed.edu 